\title{
Ventile auch bei homogenem Emphysem gut
}

\begin{abstract}
Hintergrund und Fragestellung: Endobronchiale Ventile (EBV) werden bei Patienten mit heterogener Emphysemverteilung und intakten interlobären Fissuren erfolgreich eingesetzt. Die Chartis-Messung ermöglicht vor Ventilimplantation, eine Kollateralventilation zwischen den ipsilateralen Lappen auszuschlieBen. Die implantierten Ventile führen zu einem Verschluss des emphysematösen Lappens und zu einer Volumenreduktion bis hin zur Atelektase des behandelten Lappens. Bisher waren Patienten mit homogener Emphysemverteilung aufgrund mangelnder Erfolgsdaten meist von der Ventilbehandlung und der chirurgischen Lungenvolumenreduktion ausgeschlossen.
\end{abstract}

Patienten und Methoden: Patienten in dieser prospektiv randomisierten multizentrischen Studie erhielten entweder Ventile mit komplettem Lappenver-

Originalie

Valipour A, Slebos DJ, Herth F et al. Endobronchial Valve Therapy in Patients with Homogeneous Emphysema: Results from the IMPACT Study. Am J Respir Crit Care Med. 2016 Nov 1; 194(9):1073-82. tiven Softwareanalyse der CT-Bilder erfolgte die Selektion des zu behandelnden Lappens. Ein homogenes Emphysem lag vor, wenn der Unterschied im „emphysema destruction score“ zwischen den ipsilateralen Lappen weniger als $15 \%$ betrug. In der Perfusionsszintigrafie durfte der Unterschied der Diffusion zwischen rechter und linker Lunge nicht mehr als $20 \%$ betragen. Alle Patienten erhielten eine Chartis-Messung vor Randomisierung. Nur jene mit fehlender Kollateralventilation wurden randomisiert. Es folgte ein kompletter Lappenverschluss mittels EBV.

Ergebnisse: In der Ventilgruppe verbesserte sich der primäre Endpunkt - die relative $\mathrm{FEV}_{1}$-Änderung nach 3 Monaten - von $13,7 \pm 28,2 \%$, in der Standardgruppe hingegen verschlechterte er


sich nicht signifkant. Im St George Respiratory Questionnaire Score besserte sich die Ventilgruppe um 9,6 Punkte, der 6-Minutengehtest nahm um $40 \mathrm{~m}$ zu und das RV um $480 \mathrm{ml}$ ab. Schwerwiegende unerwünschte Ereignisse traten in der Ventilgruppe in $44,2 \%$ auf, darunter $25,6 \%$ Pneumothoraces, aber auch COPD-Exazerbationen und Pneumonien. In der Kontrollgruppe kam es zu einem Todesfall aufgrund einer Pneumonie.

Schlussfolgerung: Es zeigte sich, dass nach Selektion auch bei Patienten mit homogenem Emphysem der Verschluss eines Lappens die Lungenfunktion und Lebensqualität bessern kann.

\section{- Kommentar von Dr. med. Maren Schuhmann}

\section{Verbesserung nicht so signifikant wie bei heterogenem Emphysem}

Bei fehlender Kollateralventilation sind EBV für Patienten mit heterogenem Emphysem erwiesenermaßen eine Therapieoption, um Belastbarkeit und Lungenfunktion dieser schwerkranken Patienten zu verbessern [1]. Hierzu muss ein Lappen komplett verschlossen werden, um das gewünschte Ergebnis einer Atelektase erreichen zu können. Patienten mit homogener Emphysemverteilung waren nach der NETT-Studie [2], bei der sich kein Vorteil für eine chirurgische Lungenvolumenreduktion bei homogenem Emphysem zeigte, von anderen Ventilstudien meist ausgeschlossen. Zu bemerken ist aber, dass es keine einheitliche Definition eines homogenen Emphysems gibt und die hier verwendete Definition ggf. mit verbesserter quantitative Analyse der CT-Bilder künftig erneut diskutiert werden muss. Die IMPACT-Studie ist nun die erste randomisierte multizentrische Studie, die nur Patienten mit homogenem Emphysem untersuchte. Auch diese Patienten profitierten lungenfunktionell wie auch in Belastbarkeitstests und Lebensqualität-Scores, wenn eine entsprechende Volumenreduktion erreicht wird. Jedoch ist in dieser Studie eine hohe Pneumothoraxrate zu beobachten, die meist zumindest einer Drainage, ggf. auch eine Entfernung der Ventile nötig machte. Gerade bei Patienten, die schon durch ihre Grunderkrankung schwer eingeschränkt sind, muss man dieses Risiko vor der Intervention daher gründlich abwägen. Doch sind eben bei diesen Patienten die Therapieoptionen extrem limitiert, und die Ventilimplantation sollte mit Blick auf die Ergebnisse erwogen werden.

\footnotetext{
Literatur

1. Davey C, Zoumot Z, Jordan S et al. Bronchoscopic lung volume reduction with endobronchial valves for patients with heterogeneous emphysema and intact interlobar fissures (the BeLieVeR-HIFi study): a randomised controlled trial. Lancet 2015;386:66-73.

2. Fishman A, Martinez F, Naunheim K et al. National Emphysema Treatment Trial Research Group. A randomized trial comparing lung-volume reduction surgery with medical therapy for severe emphysema. N Engl J Med 2003;348:59-73.
}

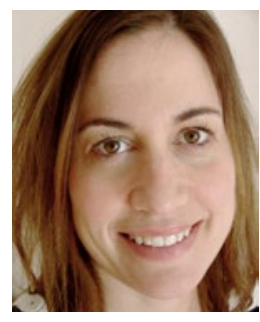

Dr. med. Maren Schuhmann

Thoraxklinik Heidelberg Röntgenstr. 1, 69126 Heidelberg maren.schuhmann@med.uni-heidelberg.de 